Proceedings of the International School and Conference on Optics and Optical Materials, ISCOM07, Belgrade, Serbia, September 3-7, 2007

\title{
Far-Infrared Study of DX-Like Centers in $\mathrm{Pb}_{0.95} \mathrm{Mn}_{0.05} \mathrm{Te}(\mathrm{Ga})$
}

\author{
D. Stojanović ${ }^{a}$, J. Trajić ${ }^{a}$, B. Hadžić ${ }^{a}$, M. RomČević ${ }^{a}$, \\ I.I. $\operatorname{IVANCHIK}^{b}$, D.R. KHOKHLOV ${ }^{b}$ AND N. ROMČEVIĆ ${ }^{a, *}$ \\ ${ }^{a}$ Institute of Physics, Pregrevica 118, 11080 Belgrade, Serbia \\ ${ }^{b}$ Moscow State University, 119899 Moscow, Russia
}

\begin{abstract}
We present far-infrared reflection spectra of $\mathrm{Pb}_{0.95} \mathrm{Mn}_{0.05}$ Te single crystal doped with gallium between 10 and $300 \mathrm{~K}$. The analysis of the far-infrared reflection spectra was made by a fitting procedure based on the model of coupled oscillators. Together with the strong plasmon-phonon coupling we obtain three local modes of gallium at about $122 \mathrm{~cm}^{-1}, 166 \mathrm{~cm}^{-1}$, and $192 \mathrm{~cm}^{-1}$. The position of these modes depends on impurity center charge, and their intensity depends on temperature.
\end{abstract}

PACS numbers: $71.28+\mathrm{d}, 78.20 .-\mathrm{e}, 63.20 . \mathrm{Pw}$

\section{Introduction}

Lead chalcogenides are well-known materials for the infrared optoelectronics. They are mainly used for the production of lasers and LEDs operating in the middle and far infrared. The band gaps energies of IV-VI semiconductors, which have direct gaps at $L$ point of the Brillouin zone, range from 0 to $0.4 \mathrm{eV}$. As the band gaps depend on composition of mixed crystals, lasers made from these materials exhibit large tuning ranges making them uniquely suited for high resolution molecular spectroscopy applications.

The problem of the DX centers in semiconductors has been a subject of intensive theoretical and experimental research in the last twenty years $[1,2]$. The basic characteristic feature of these impurity centers is a strong electronphonon interaction which causes the appearance of barriers in a configuration space between the states with different number of electrons. Doping of the lead telluride-based alloys with some impurities of the group III as In, Ga results under certain conditions in an appearance of the DX-like impurity centers providing the existence of the Fermi level pinning [1] and persistent photoconductivity effect [2] at low temperature.

*corresponding author; e-mail: romcevi@phy.bg.ac.yu 
In our earlier papers we analyzed the Raman scattering and far-infrared reflection spectra of indium and gallium doped $\mathrm{PbTe}$, pure $\mathrm{Pb}_{1-x} \mathrm{Mn}_{x} \mathrm{Te}$ and indium doped $\mathrm{Pb}_{1-x} \mathrm{Mn}_{x} \mathrm{Te}$ [3-5]. We found that the phonons in $\mathrm{Pb}_{1-x} \mathrm{Mn}_{x}$ Te show an intermediate one-two mode behavior. In III-group impurities doped PbTe based alloys we registered three impurity local modes. By extending this study to infrared reflection spectra of $\mathrm{Pb}_{1-x} \mathrm{Mn}_{x} \mathrm{Te}(\mathrm{Ga})$ single crystal samples we expect to get a complete picture of the III-group impurity states in $\mathrm{Pb}_{1-x} \mathrm{Mn}_{x} \mathrm{Te}$ and in that way clarify the behavior of the DX-like centers in these systems.

\section{Experimental}

The sample $\mathrm{Pb}_{0.95} \mathrm{Mn}_{0.05} \mathrm{Te}+0.2$ at.\% Ga single crystal was grown by the Czochralski method. The gallium impurity was introduced into the liquid zone. A Bruker 113v spectrometer with an Oxford cryostat was used to measure the far-infrared reflection spectra at low temperature.

\section{Results and discussion}

The far-infrared reflection spectra of the $\mathrm{Pb}_{0.95} \mathrm{Mn}_{0.05} \mathrm{Te}+0.2$ at.\% Ga single crystal is shown in Fig. 1. The experimental data are represented by circles. The solid line was obtained using a modified factored dielectric function model of coupled plasmon-phonon modes [6]:

$$
\begin{aligned}
\varepsilon_{\mathrm{f}}(\omega) & =\varepsilon_{\infty} \frac{\prod_{j=1}^{2}\left(\omega^{2}+\mathrm{i} \gamma_{l j} \omega-\omega_{l j}^{2}\right)}{\omega\left(\omega+\mathrm{i} \gamma_{p}\right)\left(\omega^{2}+\mathrm{i} \gamma_{t} \omega-\omega_{t}^{2}\right)} \prod_{n=1}^{p} \frac{\omega^{2}+\mathrm{i} \gamma_{L n} \omega-\omega_{L n}^{2}}{\omega^{2}+\mathrm{i} \gamma_{t n} \omega-\omega_{0 n}^{2}} \\
& \times \prod_{k=1}^{2} \frac{\omega^{2}+\mathrm{i} \gamma_{\mathrm{LO} k} \omega-\omega_{\mathrm{LO} k}^{2}}{\omega^{2}+\mathrm{i} \gamma_{\mathrm{TO} k} \omega-\omega_{\mathrm{TO} k}^{2}} .
\end{aligned}
$$

The $\omega_{l j}$ and $\gamma_{l j}$ parameters of the first numerator should be understood as the eigenfrequencies and damping coefficients of the longitudinal plasmonsphonon $(\mathrm{LP}+\mathrm{LO})$ waves, which arise as a result of the interaction of the initial modes. The parameters of the first denominator correspond to similar characteristics of the transverse (TO) vibrations; the $\gamma_{p}$ value describes the LP-mode damping coefficient in the limit of zero frequencies and $\varepsilon_{\infty}$ constant denotes the contribution of the excitations at high frequencies relative to the spectral interval of interest. The second term in Eq. (1) represents Ga-impurity local modes, to be discussed later. $\omega_{\mathrm{LO} k}$ and $\omega_{\mathrm{TO} k}$ are the longitudinal and transverse frequencies, and $\gamma_{\mathrm{LO} k}$ and $\gamma_{\mathrm{TO} k}$ stand for the damping of uncoupled modes of the host crystal.

The oscillators denoted by $j=1,2$ in Eq. (1) are the dominant structures in the far-infrared reflection spectra and represent the position of the coupled plasmon-LO phonon modes. The frequencies of these modes $\left(\omega_{l j}(j=1,2)=\omega_{ \pm}\right)$ [6] are marked by arrows in Fig. 1 . The values for $\omega_{\mathrm{LO}}$ were determined as described in Ref. [6]. At $T=300 \mathrm{~K}$, the corresponding PbTe and MnTe-like vibration modes (TO/LO pairs) in $\mathrm{Pb}_{1-x} \mathrm{Mn}_{x} \mathrm{Te}$ are $(37 / 52) \mathrm{cm}^{-1}$ and $(54 / 107) \mathrm{cm}^{-1}$, respectively, as we obtained earlier [4]. Oscillator of a weak intensity, $70 \mathrm{~cm}^{-1}$ (the 

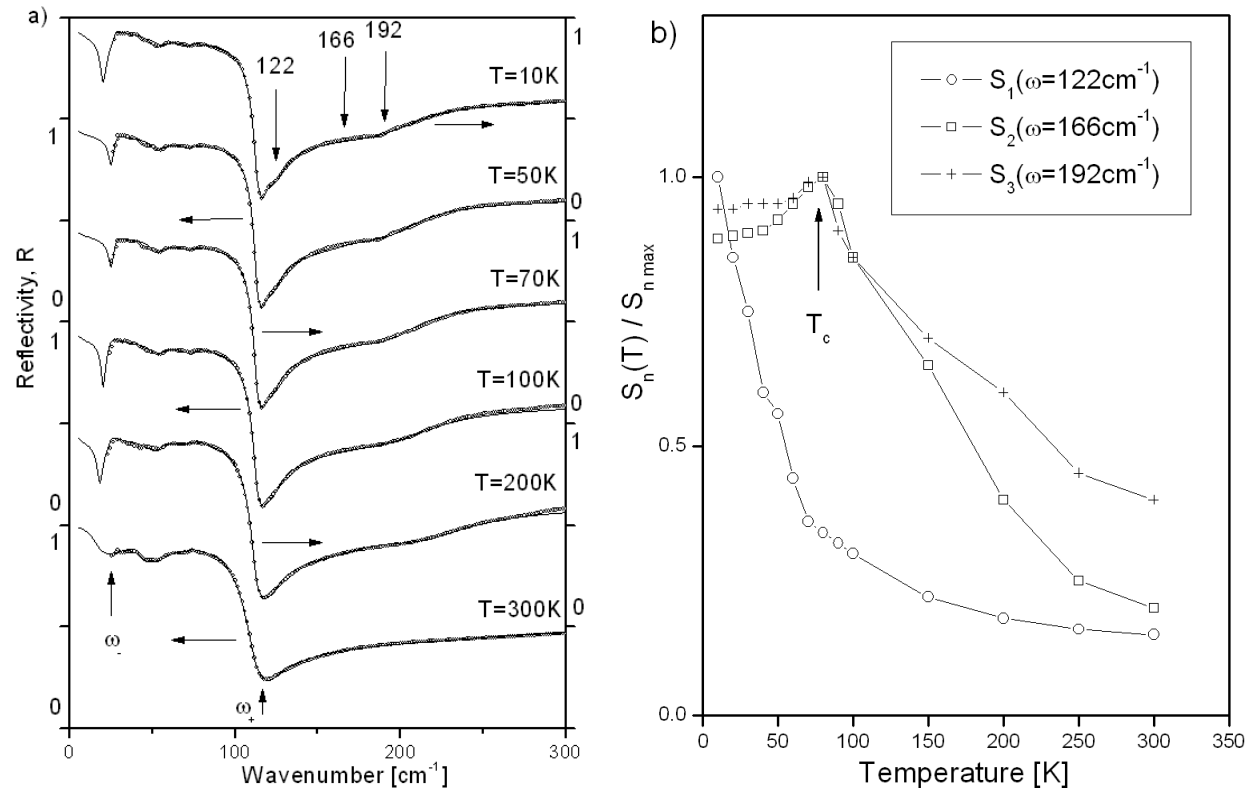

Fig. 1. (a) Far-infrared reflection spectra of $\mathrm{Pb}_{0.95} \mathrm{Mn}_{0.05} \mathrm{Te}+0.2$ at.\% Ga single crystal. Experimental spectra are represented by circles. The solid lines are the calculated spectra obtained by fitting procedure based on the model given by Eq. (1) with $p=3$. Dashed vertical lines denote the position of $\omega_{0 n}$ at 122,166 and $192 \mathrm{~cm}^{-1}$. (b) Temperature dependence of the normalized strength of the oscillators $\left(S_{1 \max }=160 \mathrm{~cm}^{-2}\right.$, $\left.S_{2 \max }=9516 \mathrm{~cm}^{-2}, S_{3 \max }=7809 \mathrm{~cm}^{-2}\right)$.

third term in Eq. (1)), belong to modes from the edge of the Brillouin zone, where the density states of PbTe have maximum on these frequencies [4]. Beside the plasmon-phonon interaction, the broad structure appears for frequencies above $\omega_{+}$at $T<200 \mathrm{~K}$. Taking into account the Raman measurements [5] and results from $\mathrm{PbTe}(\mathrm{Ga})[3]$ in Eq. (1) it was taken that $p=3$ where $\omega_{0 n}$ is characteristic frequency, $\gamma_{L n}$ and $\gamma_{t n}-$ damping, and $\omega_{L n}-$ a parameter connected to the oscillator strength $\left(S_{n} \sim \omega_{L n}^{2}-\omega_{0 n}^{2}\right)$. The characteristic frequencies $\omega_{01}=122 \mathrm{~cm}^{-1}$, $\omega_{02}=166 \mathrm{~cm}^{-1}$ and $\omega_{03}=192 \mathrm{~cm}^{-1}$ remain unchanged with the temperature change. As we see from Fig. $1 \mathrm{~b}$ the strength of oscillator at $122 \mathrm{~cm}^{-1}$ increases with lowering of temperature. The decrease in temperature up to $T=80 \mathrm{~K}$ increases the strength of oscillators at 166 and $192 \mathrm{~cm}^{-1}$. Further decrease in temperature lowers the strength value. This type of behavior is a consequence of the persistent photoconductivity effect, already seen in galvanomagnetic measurements [2]. In our opinion, the nature of additional oscillators is connected to excitations of local phonons modes in the vicinity of an impurity atom. The first fact that points to this conclusion is that the additional oscillators have frequencies above the top of optical range of $\mathrm{Pb}_{1-x} \mathrm{Mn}_{x} \mathrm{Te}\left(\omega_{\mathrm{LO}}=107 \mathrm{~cm}^{-1}\right)$. Also, if the semiconductor 
is doped with a substitution impurity [7] (in this case Ga), when substitution is made on the heavier mass (in this case $\mathrm{Pb}$ ), a lighter impurity gives two localized modes: a local mode situated above the optical band and a gap mode situated above the acoustic band but below the optical band of the host lattice.

There is a question: why does one notice three local modes of Ga? Namely, the neutral state of gallium in the lattice $\mathrm{PbTe}\left(\mathrm{Ga}^{2+}\right)$ is unstable [3] and according to equation

$$
2 \mathrm{Ga}^{2+}=\mathrm{Ga}^{+}+\mathrm{Ga}^{3+}
$$

transverse to the two-electron stable state $\left(\mathrm{Ga}^{+}\right)$, which is energy-wise more favorable than the metastable single electron $\left(\mathrm{Ga}^{2+}\right)$ and state of empty center $\left(\mathrm{Ga}^{3+}\right)$. As in case $\mathrm{PbTe}(\mathrm{Ga})$ we can draw the following conclusions: the $122 \mathrm{~cm}^{-1}$ mode corresponds to the impurity center which is in an unstable one-electron state of $\mathrm{Ga}^{2+}$, the $190 \mathrm{~cm}^{-1}$ mode corresponds to impurity centers of Ga with two electrons $\left(\mathrm{Ga}^{+}\right)$and $166 \mathrm{~cm}^{-1}$ mode corresponds to an impurity center without localized states.

\section{Conclusion}

In this paper we have shown far-infrared reflection spectra of gallium doped $\mathrm{Pb}_{1-x} \mathrm{Mn}_{x}$ Te single crystal samples at temperatures between 10 and $300 \mathrm{~K}$. In addition to the strong plasma-phonon interaction, we registered three impurity local modes of gallium. The position of these modes depends on impurity center charge. The atoms of gallium in this system show DX-like center behavior. The effect of persistent photoconductivity has been registered at $T<80 \mathrm{~K}$.

\section{References}

[1] B.M. Vul, I.S. Voronova, G.M. Kalyznaya, T.S. Mamedov, T.S. Ragimova, Pis'ma ZETF 29, 21 (1979) [JETP Lett. 29, 18 (1979)].

[2] B.A. Akimov, S.A. Beloko'n, Z.M. Dashevskii, K.N. Egorov, V.M. Lakeenkov, A.V. Nikorich, L.I. Ryabova, Fiz. Tekhn. Poluprovodn. 25, 250 (1991).

[3] M. Romčević, N. Romčević, D.R. Khokhlov, I.I. Ivanchik, J. Phys., Condens. Matter 12, 8738 (2000).

[4] N. Romčević, M. Romčević, D.R. Khokhlov, A.I. Belogorokhov, I.I. Ivanchik, W. König, Infrared Phys. Tekh. 40, 453 (1999).

[5] N. Romčević, Z.V. Popović, D.R. Khokhlov, W. Konig, Z. Phys. 104, 475 (1997).

[6] A.A. Kukharskii, Solid State Commun. 13, 1761 (1973).

[7] B.A. Volkov, V.P. Kusmin, O.A. Pankratov, Fiz. Tverd. Tela 24, 415 (1982). 